\title{
The Behaviour of Heavy Gas and Particulate Clouds
}

\author{
R. J. BETTIS, G. M. MAKHVILADZE, and P. F. NOLAN \\ Department of Chemical Engineering \\ Polytechnic of the South Bank. \\ London SE1 OAA, United Kingdom
}

ABSTRACT

Models have been applied to stages of two phase releases following the failure of pressurised vessels. The initial expansion stage has been modelled using an experimental apparatus involving the measurement of pressure histories and of Freon-11 aerosol droplet sizes and velocities. The experimental data combined with a thermodynamic analysis has allowed estimations of the time dependent processes. The analytical description of the critical pressure decrease on the opening of the vessel is presented.

The later evolution stage has been mathematically modelled by assuming that the two phases have separate velocities. The cloud motion characteristics have been shown to depend upon the degree of hydrodynamic interactions between the particles via the gaseous phase. For small particles'fractional volumes, this interaction is small and each particle behaves as a single particle corresponding to a "filtration" regime. However, for large concentrations the air between the particles becomes entrained due to the particle motion and the cloud velocity exceeds that of the single particle. In this "entrainment". regime large scale vortex motion occurs.

\section{INTRODUCTION}

Despite continuous efforts (1-9), problems still remain with regard to the prevention of explosions following the failure of pressurised storage vessels Substances, such as liquid petroleum gas (e.g. propane) require the maintenance of an increased pressure in the vessel simply because their boiling points at normal pressure are lower than ambient temperatures (e.g. boiling point of propane is $-45^{\circ} \mathrm{C}(228 \mathrm{~K})$ at $\left.1 \mathrm{bar}\right)$. In order to store propane as a 1 iquid at, say $20^{\circ} \mathrm{C}$ (293K) a pressure of 10 bar must be applied. Such storage under pressure increases the risk of explosion due to vessel failure $(1,2,5,7)$.

The failure of a vessel, is accompanied by an abrupt decrease in pressure, the liquid becomes superheated, rapidly vaporises, and a mixture of vapour and liquid is released from the vessel. The cloud formed as this release mixes with the surrounding air is highly dangerous, both from the potential environmental pollution effects and also from the possibility of a flamable mixture being ignited. Both gas dynamics and hydrodynamics are involved in a liquid petroleum gas (LPG) release.

The present address for G. M. Makhviladze is Institute for Problems in Mechanics, USSR Academy of Sciences, prosp. Vernadskogo, 101, Moscow, 117256. 
The experimental work modelled the first phase of a pressurised liquid release, during which the pressure falls to that of the surroundings. The model material was Freon-11, which has a normal atmospheric boiling point of C. $23^{\circ} \mathrm{C}(296 \mathrm{~K})$.

The release was produced by "failing" a fully instrumented vessel consisting of two hemispheres held together pneumatically and containing the model material maintained at measured internal pressure, temperature and fil1leve1. The assembled vessel is $114 \mathrm{~mm}$ in diameter, and has a maximum fill of $1.1 \mathrm{~kg}$. of Freon-11. A heater in the vessel allows energy to be put into the closed system, increasing the temperature and pressure of the Freon-11. When the appropriate release conditions had been established, the pressure on the pneumatic system holding the vessel together was reversed, and the two halves pulled apart.

The resulting release consisted of three parts:

(i) Vapour; produced during the rapid boiling as the pressure was reduced.

(ii) Aerosol; the liquid broken up during boiling and entrained into the expanding vapour, which carried it away from the release point. The size of the droplets which form the release was measured using a laser diffraction system, and flash photography. The profile of the spray, and its overall velocity was examined using video and high speed cine photography.

(iii) Bulk liquid; any liquid which remained in the immediate vicinity of the release point. This was collected on an instrumented tray below the vesse1.

Preliminary results in Table represent average values from several experiments.

The droplet sizes (the mean sizes of the best $10 \mathrm{~g}$.- normal distribution) were measured at a point level with the centre-1ine of the vessel, at a distance of $0.5 \mathrm{~m}$ from the centre. The velocity estimates were average values over the first two metres of travel away from the vessel. The time given as release time was the time for equalisation of vessel internal pressure with the atmosphere. For simplicity, in calculations of the later stage of evolution the mean droplet size was taken as $100 \mu \mathrm{m}$ and the characteristic velocity of the spray as $10 \mathrm{~m} \mathrm{~s}^{-1}$.

TABLE 1. Results from experimental work

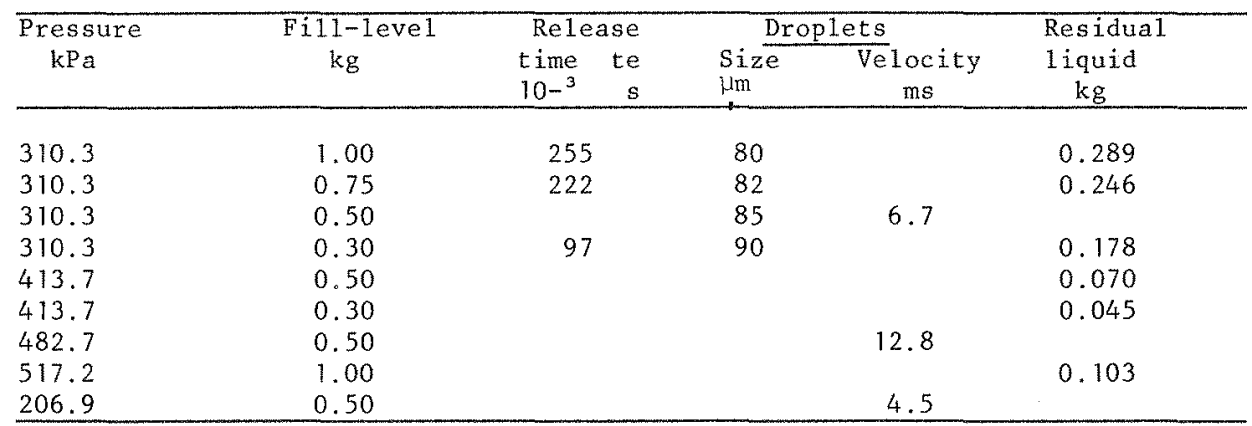


Following the example set by Hardee and Lee (1), the initial expansion can be considered isentropic

thus $S_{t}=x_{t} S_{V t}+\left(1-x_{t}\right) S_{L t}=a$ constant

hence $S_{o}=x_{0} s_{V_{0}}+\left(1-x_{0}\right) s_{L o}=x_{t} s_{V t}+\left(1-x_{t}\right) S_{L t}$

i.e. $\mathrm{x}_{t}=\left[\mathrm{x}_{0}\left(\mathrm{~S}_{\mathrm{Vo}_{0}}-\mathrm{S}_{\mathrm{Lo}}\right)+\left(\mathrm{S}_{\mathrm{Lo}}-\mathrm{S}_{\mathrm{Lt}}\right)\right] /\left(\mathrm{S}_{\mathrm{Vt}}-\mathrm{S}_{\mathrm{Lt}}\right)$

On the assumptions that the vapour behaves as an ideal gas

$$
\rho_{\mathrm{vt}}=\mathrm{P}_{\mathrm{t}} \varphi /\left(\mathrm{R} \mathrm{T}_{\mathrm{Lt}}\right)
$$

and that the vapour temperature is a constant and equal to the room temperature, the vapour volume is given by

$$
v_{v t}=x_{v t} w_{v} / \rho_{v t}
$$

Thus, if the relationships between entropy and pressure are known, then the pressure dependencies of quality, vapour density and vapour volume can be calculated. Pressure-entropy relationships were taken from curves fitted to data from standard tables (10).

The calculated data for quality is presented in Figure 1. This figure shows that between 10 and $45 \%$ of Freon-11 is vaporised during the expansion. This agrees with the results from the experimental work and that of others $(1,3)$. However, it is the time dependence of these parameters, not pressure, which is important. Hardee and Lee (1) produced a theoretical time dependence for the pressure.

A typical experimental pressure history trace is given in Figure 2 $(P=310.3 \mathrm{kPa}, W=1 \mathrm{~kg})$. Point (a) corresponds to the beginning of vesse1 failure. This is followed by a rapid fall in pressure, taking 15 to $20 \times 10^{-3} \mathrm{~s}$. It is thought that this is due to the exit of vapour from the vessel, and occurs before the flash boiling begins. Occasionally there is then a small rise in apparent pressure lasting for a similar time. This pressure is probably due to the impact of liquid on the pressure sensor resulting from the onset of flash boiling. After this "momentum peak" has subsided the pressure falls smoothly to atmospheric, although at a much slower rate than in the initial decay. It is during this period that the two phase release occurs, with the expanding vapour entraining the liquid and carrying it away from the vesse1.

It is possible to model the changes, which occur in the pressure history prior to the onset of flash boiling at Point (b).

Integrating the continuity equation, over the volume $V_{V}$, corresponding to the volume occupied by gas in the vesse1, leads to

$$
\frac{\partial}{\partial t} \int_{V_{v}} \rho_{v} d V+\int_{V_{v}} \operatorname{div} \rho_{v} \vec{u}_{v} d v=0
$$




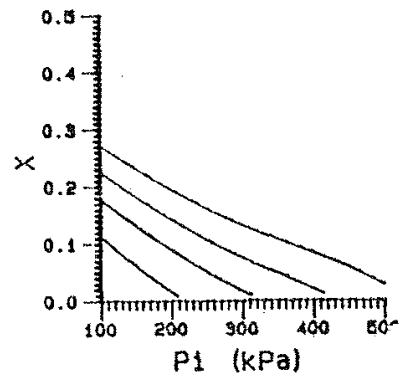

$F 111(\mathrm{~kg})=0.50$

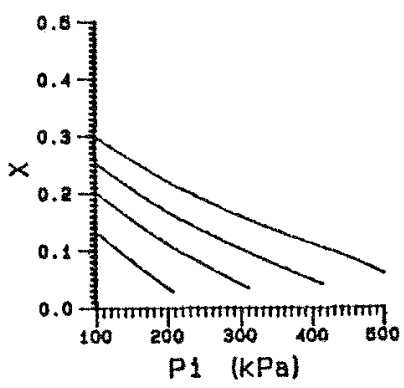

$F 121(\mathrm{~kg})=0.30$

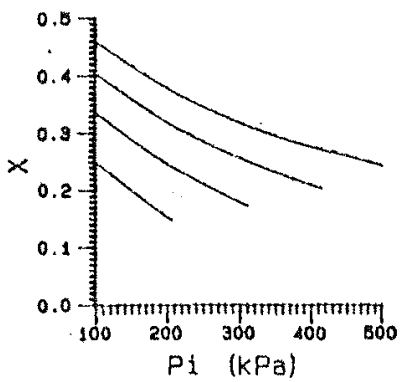

Figure 1. Theoretical quality curves for $2,3,4$ and 5 atm

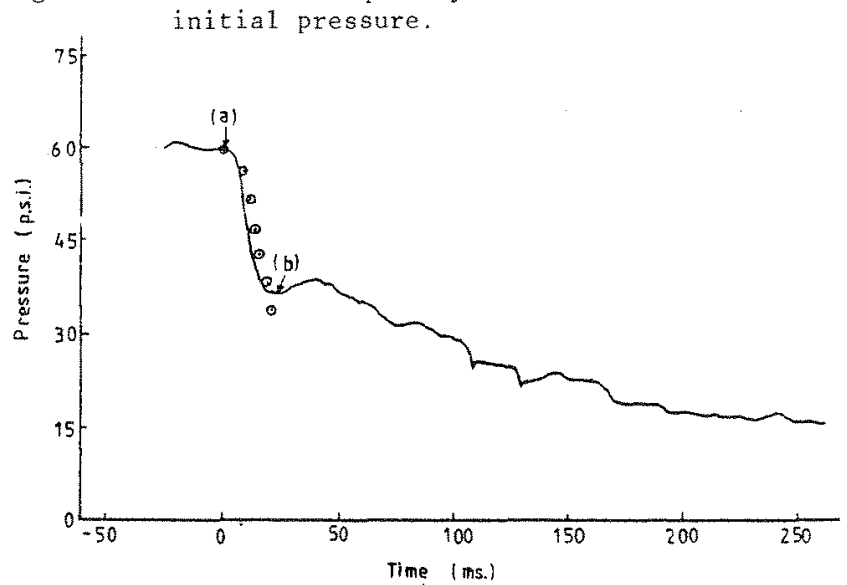

Figure 2. Pressure versus time during release. 
By applying Gaussian theory to the second term, and assuming that the gas velocity at the vessel walls is zero, and that the annular velocity, $u$, is constant, then

$$
\int_{v} \operatorname{div} \rho_{v} \vec{u}_{v} d v=\rho_{v} u^{\prime}{ }_{v} A
$$

and

$$
\rho_{\mathrm{v}}=\mathrm{Z} \mathrm{P}_{\mathrm{V}} \text { where } \mathrm{Z}=\mathrm{W}_{\mathrm{V}} \cdot /\left(\mathrm{R} \mathrm{T}_{\mathrm{V}}\right)=\text { constant }
$$

The pressure of the gas in volume $V_{v}$ can be considered constant but will change rapidly on the failure of the vessel

$\mathrm{V}_{\mathrm{v}} \mathrm{dP} / \mathrm{dt}=-\mathrm{P}_{\mathrm{v}} \mathrm{u}_{\mathrm{v}}^{\mathrm{r}} \mathrm{A}$

By taking a hydraulic approximation to the momentum balance, it is possible to introduce a quasi-stationary relationship for the out-flow of the jet

$$
u_{v}^{\prime}=\left[\left(P_{v}-P_{A}\right) / \rho_{v}\right]^{1 / 2}
$$

The annular area, A, changes during the process from zero to the value A at the time of pressure equalisation. For simplicity, this will be described by a power law

$$
A=A_{e}\left(t / t_{e}\right)^{n}
$$

By substitution of equations (10) and (11) into (9)

$$
\partial p^{\prime} / \partial t^{\prime}=\left[p^{\prime}\left(p^{\prime}-1\right)\right]^{1 / 2}\left(t^{\prime}\right)^{n}
$$

where $\mathrm{p}^{\prime}=\mathrm{P}_{\mathrm{V}} / \mathrm{P}_{\mathrm{A}} \quad \mathrm{t}^{\prime}=\mathrm{t} / \mathrm{t}^{*}$

and $t *=\left[z^{1 / 2} v_{v} /\left(A_{e} t_{e}\right)\right]^{1 /(n+1)} t_{e}$

Equation (12) describes the rate of the pressure drop. Integration of equation (12) with the initial condition of

$\mathrm{p}^{\prime}=\mathrm{p}_{0}^{\prime}$ where $\mathrm{p}_{0}^{\prime}=\mathrm{P}_{0} / \mathrm{P}_{\mathrm{A}}$

leads to

$$
\frac{\left(t^{\prime}\right)^{n+1}}{n+1}=\ln \left(\frac{2 p^{\prime}-1+\left[\left(2 p^{\prime}-1\right)^{2}-1\right]^{1 / 2}}{2 p^{1}-1+\left[\left(2 p^{\prime}-1\right)^{2}-1\right]}\right)
$$


This equation is only valid for the initial period and cannot be applied for the whole transient process. The value of $t^{*}$ in equation (13) determines the characteristic time of the pressure drop.

Fiqure 2 also illustrates the correlation between this theoretical approach and the experimental pressure drop for the following conditions

$V_{V}=0.97 \times 10^{-3} \quad \mathrm{~m}^{3} \mathrm{~W} / \mathrm{V}=137.37 \times 10^{-3} \quad T_{V}=300 \mathrm{~K} \quad t_{e}=0.229 \mathrm{~s}$.

It was assumed that

$\mathrm{n}=1 \quad \mathrm{~A}_{\mathrm{e}}=0.59 \times 10^{-3}$ (corresponding to a final opening of $1.5 \mathrm{~mm}$ )

Between points (a) and (b) in Figure 2 good agreement was found but, as expected, deviations occurred beyond this region.

MATHEMATICAL MODEL - The Later Evolution

The final stage of cloud evolution is assumed to start when the particles have reached their maximum height. Here they can be described as a cloud of stationary, mono-sized, spherical particles suspended above a horizontal surface. The particles then start to move downwards under gravity, giving rise to gas movement.

The main assumption of multi-phase mechanics is that the diameter of the particles and the mean distance between the particles are much smaller than the distance over which there is a significant change in the macroscopic parameters (11). The system can be considered as two intermixed, interacting media, the gas and the particles. The diameter of the particles is much larger than the molecular scales.

Brownian motion, evaporation and break-up of the particles are neglected, although it is recognised that these may be important, particularly evaporation.

Assuming $\theta_{\mathrm{L}} \ll \theta_{\mathrm{V}}, \rho_{\mathrm{v}} / \rho_{\mathrm{L}} \ll 1$ and constant dynamic viscosity.

The evolution of the cloud can be considered to be an isothermal process, since the heating of the system due to the viscous energy dissipation is small and both gas and air temperature are held at the initial temperature $\mathrm{T}_{0}$.

Both planar (see reference (12)) and axi-symmetrical cases can be considered. For the planar case, it is assumed that the cloud size is greater in one horizontal direction than the other. The force for interaction at the interface is defined by

$$
\overrightarrow{\mathrm{F}}=\frac{3}{4} \frac{\varepsilon}{\mathrm{d}} \mathrm{C}_{\mathrm{d}} \operatorname{Re}_{\mathrm{p}} \frac{\rho}{\mathrm{T}} \mathrm{v}-\rho_{\mathrm{O} o}^{\mathrm{L}}-\left|\overrightarrow{\mathrm{u}}_{\mathrm{v}}-\overrightarrow{\mathrm{u}}_{\mathrm{L}}\right| \quad\left(\overrightarrow{\mathrm{u}}_{\mathrm{v}}-\overrightarrow{\mathrm{u}}_{\mathrm{L}}\right)
$$

$$
\text { where } \begin{aligned}
C_{d} & =\left(1+0.158 \operatorname{Re}_{p}^{0.5}\right) 24 / \operatorname{Re}{ }_{p} \\
\operatorname{Re}_{\mathrm{p}} & =\operatorname{Re}{ }_{p o}\left|\vec{u}_{\mathrm{v}}-\vec{u}_{\mathrm{L}}\right| \rho_{\mathrm{v}} / \Theta_{\mathrm{v}}
\end{aligned}
$$

The origin for the co-ordinates is situated under the cloud mass centre, the $x$-coordinate being along the surface and the $y$-coordinate along the plane of symmetry for the planar case and along the axis of symmetry for the axisymmetrical case. 
Initially, the stationary gas is in a static equilibrium with the cloud of stationary particles and a Gaussian distribution of particle concentration about the centre is applicable. The boundary conditions take into account the symmetry about the plane $x=0$; and the equilibrium state at infinity.

Particles which reach the surface play no further part in the process.

In the above example, the following values for the parameters are based on data from the initial expansion stage

$$
\begin{aligned}
& \mathrm{k}=10^{3} \mathrm{~cm}^{-3} \\
& \gamma=1.4 \\
& { }_{\text {LO }}=10^{-3}=10^{-3} \\
& \mathrm{M}^{2}=0.72 \times 10^{-3}
\end{aligned}
$$

and the terms which depend on particle size have ranges

$$
\begin{aligned}
& d=3.3 \times 10^{-6} \rightarrow 2 \times 10^{-4} \\
& \operatorname{Re}_{\text {po }}=6500000 \mathrm{~d}=21.4 \rightarrow 1300
\end{aligned} \quad \operatorname{Re}=7 \rightarrow 60 \Theta_{\mathrm{LO}} \simeq 10^{-3} \rightarrow 10^{-2} \quad \mathrm{~h}=1.5 \rightarrow 14
$$

The external Reynolds' numbers are large and indicate turbulent motion and are estimated using an effective turbulent viscosity. The particles are very small in comparison with the scale of turbulence and the Reynolds' number for the particles uses a molecular viscosity. Numerical methods have been developed (13) and two regimes of cloud evolution can be considered.

The interaction with the horizontal surface begins when the height of the cloud centre is equal to or approaching twice the radius of the cloud.

The stationary particles start to move downwards under gravity. The entrainment of the surrounding gas due to friction is described by an empirical law (equation (15)). The effect of entrainment increases with decreasing distance between the particles. In this case hydrodynamic interaction occurs between the particles throughout the cloud as a result of large scale motion of the air. The velocity of the cloud increases continuously and exceeds that of a single particle. This is called the "entrainment" regime.

If the distance between the particles is sufficient, each particle behaves as a single free-falling sphere because it is unaffected by gas motion caused by the presence of others. This is called the "filtration" regime; the gas filters through the particles.

The boundary between the two regimes in the planar case is described by

$$
\ln \Theta_{\text {LO }}=\left(0.825 \times 10^{4}\right) \mathrm{d}-4.56
$$

with $7 \%$ accuracy for the ranges

$$
3.310^{-5} \leq \mathrm{d} \leq 210^{-4} \text { and } 10^{-5} \leq \theta_{\text {Lo }} \leq 10^{-2}
$$

At a fixed value of $d$, the filtration regime occurs if the parameter $\theta_{\text {if }}$ is small. In the filtration regime, as every particle moves individually, the initial cloud shape does not change. In the entrainment regime the cloud shape changes continuously. At first, the lines of equal particle concentration form concentric circles. The cloud is similar to a "drop" moving through a liquid i.e. the particles move downwards in the centre of the cloud and move upwards near the edge. With time and an increasing cloud velocity, the "drop" is 

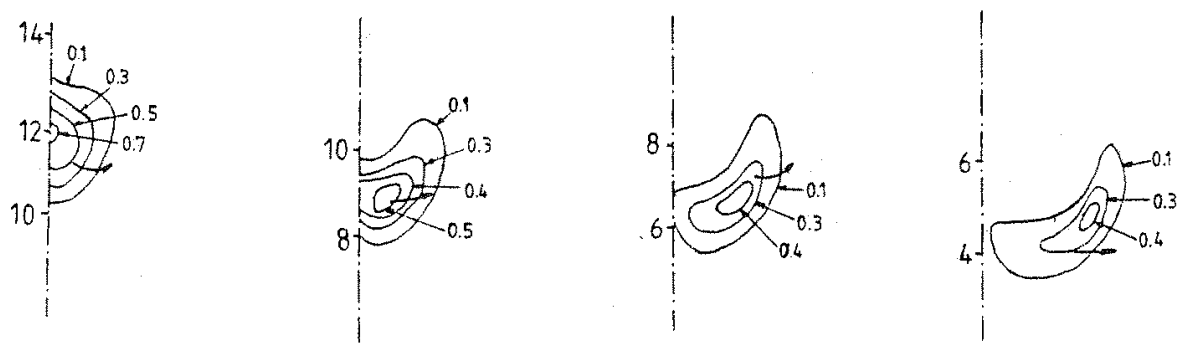

Figure 3. Cloud evolution with high particle concentration.
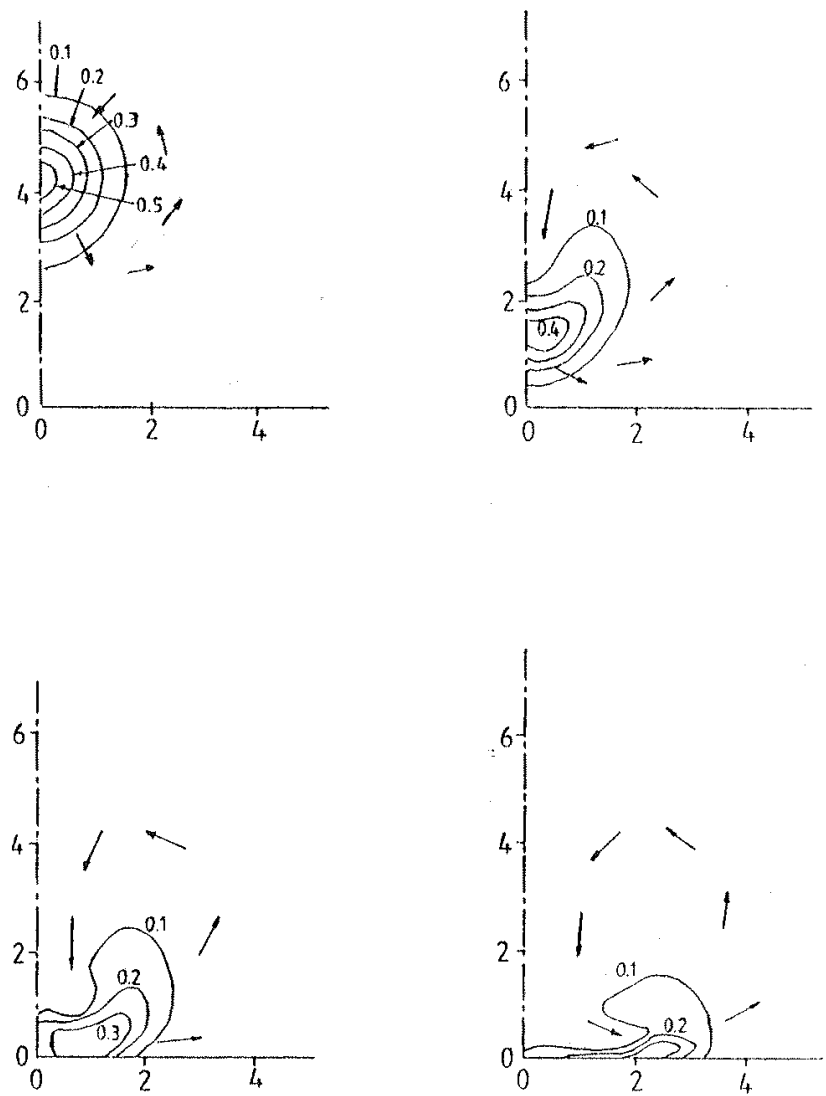

Figure 4. Characteristics of cloud sedimentation. 
deformed. A "dimple" forms in the bottom of the "drop". Gradually the drop transforms into the "cap" described previously (14, 15, 16). The gas zone is increasing continuously. Large scale vortex motion, causing lateral displacement of the particle, is formed. The zones with maximum particle concentration are removed from the plane $x=0$ (or at axis $x=0$, in the axisymmetrical case). As a result, the lines of equal concentration breakoff at a definite time.

Two symmetrical cylindrical vortices with the particles in their core are formed in the planar case (or toroidal vortex in the axi-symmetrical case). The evolution of the cloud is shown in Figure 3. The flow field and lines of equal concentration are presented for $\theta_{0}=10^{-3}$ and $\mathrm{d}=6.67 \times 10^{-5}$ at various times i.e. (a) 4.16 (b) 6.94 (c) 9.71 (d) 12.5

The process of evolution of the cloud can be followed experimentally by noting the changes in shape.

\section{CHARACTERISTICS OF SEDIMENTATION}

In the filtration regime all the particles are descending vertically on direct line trajectories. The interaction obviously does not depend on the initial cloud height.

The distribution of the particle concentration falling on the surface can be determined analytically by means of integration of the initial particle distribution on coordinate $\mathrm{y}$.

In the entrainment regime the large scale vortex motion leads to lateral displacement of the particles above the surface. Therefore, the particles are scattered across the surface. The scattering increases with increasing initial cloud height.

Cloud sedimentation, using $\theta_{\mathrm{L}}=10^{-3}, \mathrm{~d}=6.67 \times 10^{-5}$ and $\mathrm{h}=4.8$ at various times are shown in Figure 4 . It is possible to identify the formation of

(a) the vortex flow structure

(b) the "cap"

(c) the deformation of cloud shape during sedimentation.

(d) gas propagation across the surface and the scattering of particles.

Initially the particles land near the line of symmetry. Then the fall-out takes place at a certain distance. The final distribution gives a maximum located at a certain distance from the central line and a considerable number of particles can fall on the surface outside the initial projection of the cloud.

This effect can be estimated by introducing a coefficient for cloud scattering onto the surface. This coefficint is equal to the fraction of the particles which land outside the initial geomtrical shadow of the cloud on the surface.

$\mathrm{e}=1-\mathrm{N}_{\mathrm{s}} / \mathrm{N}$

where $\quad N=$ total number of particles

$\mathrm{N}_{\mathrm{s}}=$ number of particles landing outside

the initial cloud projection. 
In the "filtration" regime, the coefficient is near to zero. In the "entrainment" regime, the coefficient depends on several parameters e.g. h, d and $\theta_{1}$. With increasing initial cloud height the coefficient increases, since the vortex motion takes place in a larger gas volume, and the particles move considerable distances from the line, $x=0$. For constant $h$ and $\theta$, the scattering coefficient diminishes with increasing particle size. If $\vec{\theta}^{\circ}$ is increased, keeping the other parameters constant, the coefficient "e" increases to mark the transformation from the "filtration" regime to the "entrainment" regime. It then declines because of the large vertical component of the particle velocity. The particles fall too rapidly to move from the line of symmetry.

An isothermal mixture has been assumed, since the boiling point of Freon-11 at normal pressure is not significantly different to ambient temperature. However, for other hydrocarbons the temperature difference in liquid and gaseous phases may be significant.

\section{CONCLUSIONS}

Physical and mathematical modelling has been used to investigate the separate stages of the release of a two-phase mixture from a pressurised vesse1.

In order to study the first stages of such processes, experiments have been conducted on the "failure" of a vessel filled with liquid Freon-11. Data was obtained of the size and velocity of particles, pressure equalisation time and the time dependence of the pressure in the vessel. An analysis of the experimental data combined with thermodynamic data has allowed a definition of the time dependence of the process.

The assumption that the process is isentropic, despite only approximating to the actual nature of the process, provides satisfactory results. The results obtained can be used to estimate the parameters of the cloud formed during the failure of a pressurised vessel.

Further work will involve the investigation of

(a) flow patterns and temperature distributions set up during the release, for both the particles and the vapour.

(b) the cloud formation with simultaneous combustion inside the vessel.

The mathematical formulation of the cloud evolution has been based on the two phases having separate velocities.

The character of the cloud motion has been shown to depend upon the extent of the hydrodynamic interaction which occurs between the particles via the gaseous phase.

For a low concentration of particles this interaction is small, and each particle behaves as a single particle (the "filtration" regime). If the concentration of the particles is high enough the air between the particles is entrained by the particle motion; as a result the cloud velocity exceeds that of a single particle. In this "entrainment" regime large scale vortex motion arises: the cloud is transformed into two cylindrical vortices (in the case of planar symetry). The regime of cloud motion defines the features of the sedimentation of the particles on the horizontal surface. In the "entrainment" regime, the particles entrained into the vortex motion move laterally, therefore they sediment over some distance. 


\section{Further developments will consider}

(a) The evaporation of the particles, and the inclusion of the temperature difference between the gas and the particles.

(b) The combustion of the cloud above the vessel. Methods exist to enable non-isothermal conditions to be considered $(17,18)$.

\section{NOMENCLATURE}

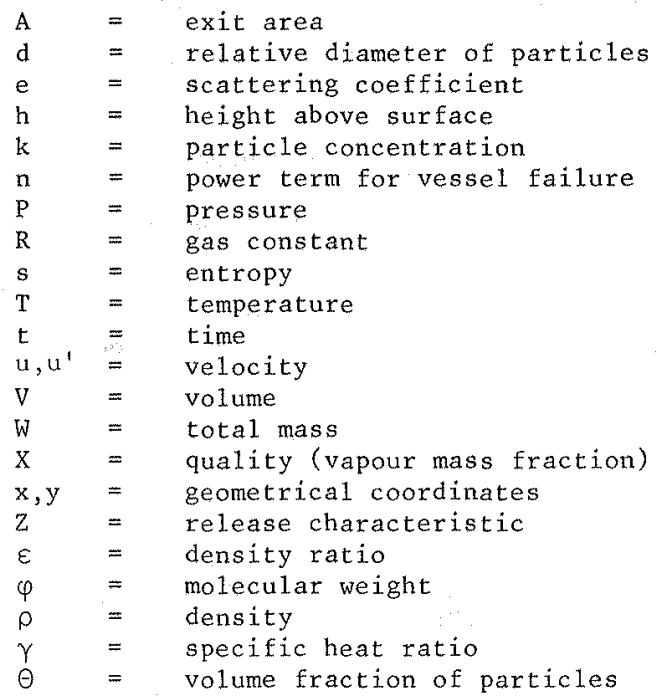

\section{Groups}

\section{Subscripts}

$\mathrm{C}_{\mathrm{F}}=$ drag coefficient
$\mathrm{M}=$ drag force
$\mathrm{p}^{\prime}=$ Mach number
$\mathrm{Re}=$ Ron-dimensional pressure
$\mathrm{t}^{\prime}=$ non-dimensional time
$\mathrm{t}^{*}=$ characteristic equalisation time

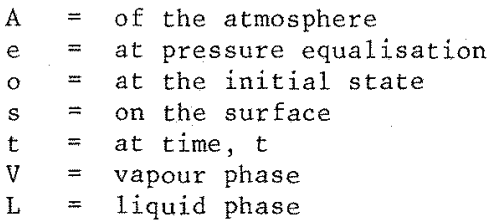

A vector quantity is indicated with the symbol "tr" above it e.g. $\vec{u}$ and div. is a vector operation.

\section{REFERENCES}

1. H.C. Hardee, D.O, Lee; "Expansion of Clouds from Pressurised Liquids" Accident analysis and Prevention, $7,91-102,1975$

2. J.A. Barton, P.F, Nolan; "Runaway Reactions in Batch Reactors" I. Chem.E. Symp. Series No. 85, The Protection of Exothermic Reactors and Pressurised Storage Vessels 13-22, Chester, 1984 
3. H. Giesbrecht, K. Hess, et a1; "Explosion Hazard Analysis of Inflammable Gas Released Spontaneously into the Atmosphere", Chemie Ingenieur Technik $52,2,114-122$, Feb. 1980

4. K. Sato, K. Hasegawa; "Study on the Fireball following Steam Explosion of of n-Pentane". 2nd Int. Symp. on Loss Prevention and Safety Promotion in the Process Industries, 1977.

5. R.E. Britter, R.F. Griffiths; "The Role of Dense Gases in the Assessment of Industrial Hazards", Journal of Hazardous Materials 6, 3-12, 1982

6. P. Field; "Dust Explosion Protection", Journal of Hazardous Materials $8,223-238,1984$

7. F.P. Lees; "Loss Prevention in the Process Industries", Butterworths, 1980

8. D.R. Blackmore, M.N. Herman, J.L. Woodward; "Heavy Gas Dispersion Models", Journal of Hazardous Materials, 6, 107-128, 1982

9. J,L Woodward, J.A. Havens, W.C. McBridge, J.R. Taft; "A Comparison with Experimental Data of Several Models for Dispersion of Heavy Vapour Clouds", Journal of Hazardous Materials, $6,161-180,1982$

10. Kuzman Rasnjevic; "A Handbook of Thermodynamic Tables and Charts", 1980

11. R.I. Nigmatulin; "Osnowy Mechaviki Getterogennych Sred. M. Nauka", 1978

12. G.M. Makhviladze, O.I. Melichov; "On the cloud Motion and Sedimentation under Gravity above a Flat Horizontal Surface" Izvestia of USSR Academy of Sciences, Liquid and Gas Mechanics, 6, 64-73, 1982

13. G.M. Makhviladze, S.B. Tscherbak; "Numerical Method for the Investigation of Non-stationary Spatial Motions of Pressurised Gas "Inginere Physical Journal, $38,3,528-537,1980$

14. G.W. Slack; "Sedimentation of Compact Clusters of Uniform Spheres", Nature, $200,4905,466-467,1963$

15. G.W. Slack; "Sedimentation of a large Number of Particles as a Cluster in Air", Nature 200, 4913, 1306, 1963

16. K. Adachi, S. Kiriyama, N. Yoshioka; "The Behaviour of a Swarm of Particles Moving in a Viscous Fluid", Chemical Engineering Science, 33, 1, 115-121, 1978

17. G.M. Makhviladze, O.I. Melichov; "On the Motion and Evolution of Cloud with Initially Hot Particles", Doklady USSR Academy of Sciences, 267, 4, $844-847,1982$

18. G.M. Makhviladze, O.I. Melichov; "Combustion of Aerosol Cloud above a Flat Horizontal Surface", Chemical Physics, 7, 991-998, 1983 